\title{
Modeling the Human Knee for Assistive Technologies
}

\author{
Massimo Sartori ${ }^{*}$ [Member, IEEE], \\ Institute of Biomedical Engineering, National Research Council, 35127 Padova, Italy; Department \\ of Information Engineering, University of Padova, 35127 Padova, Italy
}

\author{
Monica Reggiani [Member, IEEE], \\ Department of Management and Engineering, University of Padova, 36100 Padova, Italy \\ (monica.reggiani@unipd.it)
}

Enrico Pagello, and Institute of Biomedical Engineering, National Research Council, 35127 Padova, Italy; Department of Information Engineering, University of Padova, 35127 Padova, Italy (epv@dei.unipd.it)

\section{David G. Lloyd}

Centre for Musculoskeletal Research, Griffith Health Institute, Griffith University, Brisbane, Qld. 4222, Australia; School of Sport Science, Exercise and Health, The University of Western Australia, Crawley, W.A. 6009, Australia (david.lloyd@griffith.edu.au)

\section{Abstract}

In this paper, we use motion capture technology together with an EMG-driven musculoskeletal model of the knee joint to predict muscle behavior during human dynamic movements. We propose a muscle model based on infinitely stiff tendons and show this allows speeding up 250 times the computation of muscle force and the resulting joint moment calculation with no loss of accuracy with respect to the previously developed elastictendon model. We then integrate our previously developed method for the estimation of 3-D musculotendon kinematics in the proposed EMG-driven model. This new code enabled the creation of a standalone EMG-driven model that was implemented and run on an embedded system for applications in assistive technologies such as myoelectrically controlled prostheses and orthoses.

\section{Keywords}

Assistive technologies; electromyography (EMG); knee joint; musculoskeletal modeling

\section{Introduction}

THE human musculoskeletal system provides form, support, and stability to the body, with the ability to perform challenging tasks. These include 1) adapting body motion to unknown environments; 2) performing different locomotion modes conveniently switching from one to another; 3) adding compliance to joint motion; and 4) minimizing the metabolic energy consumption while moving. Understanding the musculoskeletal mechanisms underlying this human's unique ability of moving is crucial for the development of advanced assistive technologies.

\footnotetext{
(C) 2012 IEEE

*massimo.srt@gmail.com.
} 
The contribution of musculoskeletal tissues to dynamic movement has been studied using neuromusculoskeletal models driven by experimentally measured EMG signals. This has the advantage of predicting muscle behavior with no need for defining subject/task-specific objectives functions which cannot be validated experimentally. Furthermore, since EMGs occur prior to muscle force generation, this allows the resultant joint moments to be estimated before the person's intended movement occurs. To this end, researchers in biomechanics have developed sophisticated EMG-driven musculoskeletal models (EMGdriven models), but these are limited by considerable computational times [1]-[3].

Simplified versions of these models have been used for addressing real-time constraints in assistive technologies. In [4], an EMG-driven model was used to predict the passive elastic force produced by muscles and the resulting stiffness in the knee joint for the subsequent control of a transfemoral prosthetic leg. In [5]-[7], simplified EMG-driven models of lower and upper limbs were used to implement EMG-driven proportional control strategies in powered orthoses. However, the simplifications underlying these EMG-driven models significantly limit the ability of predicting muscle and joint dynamics. This may lead to less intuitive human-machine interfaces (HMIs) and to a narrower set of motor tasks to be supported by the assistive device.

In this paper, we use a musculotendon unit (MTU) model to simulate the kinematics and dynamics of muscle fibers in series with tendons. We then show that using a MTU model based on infinitely stiff tendons [8], [9] it is possible to speed up the estimation of the MTU dynamics, with respect to more sophisticated MTU models based on elastic tendons [1]-[3], while predicting similar MTU force estimates with no loss of joint moment prediction accuracy. Furthermore, we show that the identification of the model parameters leads to similar results for both the MTU models based on the infinitely stiff tendon and nonlinear elastic-tendon models. Our proposed simplification may be justified since the tendon is normally very stiff, with a strain of $3.3 \%$ when the MTU generates the maximum isometric force [10]. We then use our previously developed method ${ }^{1}$ [11] to accurately estimate the MTU 3-D kinematics and show this can be integrated with the infinitely stiff-tendon model with no additional loss of computational speed and joint moment prediction accuracy.

The novelty is this methodology results in an EMG-driven musculoskeletal model of the human knee joint that is as accurate as the sophisticated state-of-the-art models [1]-[3] but can be calibrated and executed at significantly higher computational speeds. Our proposed model can therefore be run standalone on an embedded system with limited computational power that could be used for the EMG-based control of powered orthoses or prostheses. The ability of our proposed model to convert the coordinated activation of 13 lower extremity muscles into knee joint flexion-extension moments allows obtaining an accurate prediction of the user's effort during dynamic movement. This allows implementing torque-driven controllers in which EMG signals can be used to actuate knee joint powered orthoses and prostheses in proportion to the predicted user's effort [5]-[7]. Furthermore, as demonstrated in [7], the predicted human muscle-generated joint moment could be conveniently amplified to account for situations in which the operator's muscles cannot produce enough moment around a given joint and define the motor support level the device will provide the user with. In this scenario, it becomes crucial to be able to reliably convert EMG activity into net joint moments. The ability of our proposed standalone EMG-driven model to execute one computational loop in less than $10 \mathrm{~ms}$ allows converting EMG signals into joint moments within the muscles electromechanical delay (EMD) [12]. This would allow synchronizing the human muscle contraction to the device actuation enabling the device to become a natural extension of the subject's neuromuscular skeletal system [5]-[7].

\footnotetext{
${ }^{1}$ Code and documentation available from: http://code.google.com/p/mcbs
} 


\section{Workflow}

The procedure entailed three steps: 1) collecting human movement data using motion capture technology; 2) modeling and simulating the recorded human movement; and 3) calibrating and executing the EMG-driven model.

\section{A. Human Movement Data}

Experimental movement data were collected and processed to enable the EMG-driven model calibration and subsequent execution. Six healthy male subjects (mass: $81.73 \pm 16.4 \mathrm{~kg}$, height $175.75 \pm 6.39 \mathrm{~cm}$, and age: $30.67 \pm 7.84$ years) gave their informed, written consent prior to data collection. The project was approved by the Human Research Ethics Committee at the University of Western Australia.

The motion data acquired from the subjects were static anatomical poses and dynamic gait trials. All these trials were recorded using a seven-camera Vicon MX Motion Analysis System (Oxford Metrics, Oxford, U.K.) sampling at 100Hz. During the gait trials, ground reaction forces (GRF) and EMG data were acquired at $2000 \mathrm{~Hz}$ synchronously with motion data using an in-ground force plate (AMTI, Watertown, MA) and a 16-channel acquisition system (Delsys, Boston, MA). Raw EMGs were collected from the muscles crossing the knee (see Section II-C) and were then bandpass filtered (30-450 Hz), full-wave rectified, and low-pass filtered $(6 \mathrm{~Hz})$. For each muscle, the processed EMG linear envelope was normalized against the maximum value extracted from all motion trials' EMG linear envelopes. Marker trajectories and GRFs were both low-pass filtered with the same fourthorder Butterworth filter. Cutoff frequencies were obtained performing a residual analysis on each trial [13] and ranged between 2 and $8 \mathrm{~Hz}$.

From the motion data collected, two distinct datasets were created: one for the model calibration and the other for the model validation. The calibration dataset was used to calibrate the EMG-driven model for each individual. This included data from one static trial, two walking trials, and two running trials per subject. The other dataset was used for the subsequent model validation and included ten novel walking trials $(1.4 \pm 0.3 \mathrm{~m} / \mathrm{s})$ and three novel running trials $(2.6 \pm 0.4 \mathrm{~m} / \mathrm{s})$ per subject. None of the data used for the model calibration were used in the model validation step.

\section{B. Movement Modeling}

The freely available musculoskeletal geometry modeling software OpenSim ${ }^{2}$ [14] was used to scale a generic musculoskeletal geometry model ${ }^{3}$ to each subject individually to match their anthropometry based on the experimentally measured marker positions from the static poses. During the scaling process, virtual markers were created and placed on the musculoskeletal geometry model based on the position of the experimental markers. The OpenSim inverse kinematics (IK) algorithm [14] solved for joint angles that minimized the least-squared error between experimental and virtual markers. The joint moments that needed to track the IK-generated angles were obtained using inverse dynamics and residual reduction analysis (RRA) [14]. The joint moments produced by this pathway are called "the experimental" moments. The alternate pathway to estimate joint moments was by the EMGdriven model.

\footnotetext{
${ }^{2}$ OpenSim 2.2.0 available from: https://simtk.org/home/opensim

3 Available from: https://simtk.org/home/nmblmodels
} 


\section{EMG-Driven Model}

The EMG-driven model comprises of five main components (see Fig. 1): muscle activation, musculotendon kinematics (MTK), musculotendon dynamics, moment computation, and model calibration.

In the muscle activation component [see Fig. 1(a)], EMG linear envelopes were processed using a critically damped second-order recursive filter to model the muscle's twitch response to the EMG onset and further adjusted to account for the nonlinear EMG-to-force relationship [2].

The MTK component [see Figs. 1(b) and 2 was our developed three-stage method [11] to estimate MTU length $\ell^{2 t}$ and moment arms $r$ as a function of IK-generated angles. In the first stage, a set of $\ell^{2 n t}$ nominal values were initially established for each MTU using the scaled OpenSim musculoskeletal geometry model. In the second stage, the $\ell^{\text {pht }}$ nominal values were used to calculate the coefficients of multidimensional cubic B-splines for each MTU. In the third stage, the MTU-specific spline functions were used to estimate instantaneous $\ell^{21 t}$ as a function of IK-determined angles during the EMG-driven model execution. MTU moment arms $r_{\vartheta_{i}}$ with respect to a specific joint angle $\vartheta_{\mathrm{i}}$ were obtained by the partial differentiation of the $d$-dimensional $\ell^{m t}\left(\vartheta_{1}, \ldots, \vartheta_{d}\right)$ spline function (see Fig. 2):

$r_{\vartheta_{i}}=\frac{\partial \ell^{m t}\left(\vartheta_{1}, \ldots, \vartheta_{d}\right)}{\vartheta_{i}}$. The musculoskeletal geometry model included the 13 MTUs crossing the knee joint: semimembranosus (sm), semitendinosus (st), biceps femoris long head (bflh), biceps femoris short head (bfsh), sartorius (sar), tensor fascia latae (tfl), gracilis (gra), vastus lateralis (vl), vastus medialis (vm), vastus intermedius (vi), rectus femoris (rf), medial gastrocnemius (mg), and lateral gastrocnemius (lg). For the proper estimation of MTU kinematics (i.e., $\ell^{n t}$ and $r$ ), the degrees of freedom (DOFs) of all the lower extremity joint angles were taken into account, since some of the MTUs crossing the knee also cross the hip or ankle joints. The DOFs included hip flexion-extension, hip adduction-abduction, hip internal-external rotation, knee flexion-extension (KFE), ankle plantar-dorsiflexion, ankle adduction-abduction, and ankle internal-external rotation.

In the musculotendon dynamics component [see Fig. 1(c)], the MTU force $F^{m t}$ was calculated using a Hill-type muscletendon model that included fibers and tendons in series with each other's as described previously [2]. Briefly, the fiber was modeled as parallel passive and active components, with the total fiber force $F^{m}$ given by

$$
F^{m}=\left(F_{A}+F_{P}\right) \cdot F_{o}^{m}
$$

where $F_{P}=f_{P}\left(\tilde{l}^{m}\right)+d^{m} \cdot \tilde{v}^{m}$ was the resistive passive force produced in response to fibers stretching and compression, $F_{A}=f_{A}\left(l^{m}\right) \cdot f_{V}\left(\tilde{v}^{m}\right) \cdot a(t)$ was the active force in response to fibers activation $a(t)$, and $F_{o}^{m}$ was the maximum isometric force developed at optimal fiber length $l_{o}^{m}$. The term $\tilde{l}^{m}$ was the fiber length normalized with respect to the optimal fiber length $l_{o}^{m}$, while $\tilde{v}^{m}$ was the fiber velocity normalized with respect to the maximum fiber contraction velocity $v^{\mathrm{MAX}}$. The term $d^{m}$ was the passive parallel damping element that was added to prevent any singularity of the mass-less model when activation or isometric force is zero [2]. The terms $f_{A}\left(\tilde{l}^{m}\right), f_{P}\left(\tilde{l}^{m}\right)$, and $f_{V}\left(\tilde{v}^{m}\right)$ defined how fiber force varied as a function of $\tilde{l}^{m}$ and $\tilde{v}^{m}[2]$. The tendon force $F^{t}$ was defined by a nonlinear force-strain function $f_{P}^{t}\left(\varepsilon_{t}\right)$ normalized to $F_{o}^{m}$. The term $\varepsilon_{t}=\frac{l^{t}-l_{s}^{t}}{l_{s}^{t}}$ was the tendon strain, i.e., the percentage of tendon 
length variation with respect to its slack length $l_{s}^{t}$, where $I^{t}$ was the tendon length. Using biomechanical parameters from [15], $F^{m t}$ was given by

$$
F^{m t}=F^{t}=F^{m} \cdot \cos (\phi)
$$

where $\varphi$ was the pennation angle, i.e., the angle of orientation of the fibers with respect to the tendon. Muscle fiber lengths were calculated by forward integration of fiber velocities using a Runge-Kutta-Fehlberg algorithm. ${ }^{4}$ The method presented in [16] was used to estimate initial muscle fiber length and velocities. At each time frame, fiber length was determined so that dynamic equilibrium with the tendon force was guaranteed and a single solution to (2) was provided. This integration process is time consuming since the MTU functions are inherently stiff. To reduce the computational time in this current study, an alternative method was developed based on the use of an infinitely stiff-tendon model. In this model, $F^{m t}$ depended on $I^{m}$ only because $I^{t}$ was fixed to its slack length $l_{s}^{t}$. Therefore, $F^{m}$ (and $F^{m t}$ ) could be simply calculated using (1), since instantaneous values of $P^{m t}$ and $a(t)$ were known, and if $I^{t}$ was constant, then $I^{m}$ was given by

$$
l^{m}=\sqrt{\left(l_{0}^{m} \cdot \sin \left(\phi_{0}\right)\right)^{2}+\left(l^{m t}-l_{s}^{t}\right)^{2}}
$$

where $\varphi_{0}$ was the pennation angle at optimal fiber length $l_{o}^{m}$, and $v^{m}$ was found by differentiating (3) with respect to time. In (3), the degree of MTU activation and the MTU force-length characteristics were coupled together by accounting for the change in $F_{o}^{m}$ as a function of $a(t)$ [17]: $l_{o}^{m}(t+1)=l_{o}^{m}(t)(\gamma(1-a(t))+1)$, where $\gamma$ was the percentage change in optimal fiber length which was set to $15 \%$ [2]. In this paper, we will refer to our proposed muscle-tendon model as the stiff-tendon Hill-type muscle (SHM) component and to the previous one as the elastic-tendon Hill-type muscle (EHM) component.

In the moment computation component [see Fig. 1(d)], the net KFE moments $M_{\mathrm{KFE}}$ were estimated from $F^{m t}$ multiplied by the MTU KFE moment arms $\left(r_{\mathrm{KFE}}\right)$ and summed: $M_{\mathrm{KFE}}=\Sigma_{i} \quad F_{i}^{m t} \cdot r_{\mathrm{KFE}, i}$

The model calibration component [see Fig. 1(e)] determined the values for a set of muscle tendon and muscle activation parameters that vary nonlinearly across subjects and cannot be determined experimentally or from literature [1]. Parameters were constrained to vary within predefined boundaries to ensure that MTUs always operate within their physiological range. In the muscle activation component, two global (i.e., it applies to all MTUs) activation filtering coefficients $(C 1$ and $C 2$ ) were varied between -1 and 1 to realize a stable positive solution for the critically damped recursive filter. Also, one global activation shape factor parameter was altered between -5 and 0 to account for the nonlinear EMG-to-force relationship [2].

In the musculotendon dynamics component, three strength coefficients were varied from 0.5 to 2.0 and were used to scale $F_{o}^{m}$ to account for well-documented individual differences in muscle strength. To maintain relative strength across all muscles, strength coefficients grouped MTUs into knee extensors, knee flexors, and gastrocnemius muscles [2]. Values for $l_{S}^{t}$ and $l_{o}^{m}$ were individually obtained for each MTU and subject using the methodology

\footnotetext{
${ }^{4}$ The RKF algorithm used a variable integration step size $h$ initially set to $h=T_{S} / 100$ where $T_{S}$ was the time between two kinematic samples (see Section II-A) and also represented the maximum step allowed. The $h$ step was dynamically increased or decreased during the integration as a function of the solution error.
} 
presented in [18]. Then, $l_{S}^{t}$ were further adjusted so that $l_{s}^{t}=$ initial value $\pm 5 \%$ [15]. Starting with the uncalibrated parameter set, a simulated annealing algorithm ${ }^{5}$ adjusted the parameter values to ensure that the EMG-driven model tracked the experimental $M_{\mathrm{KFE}}$ during the calibration trials, where it minimized the objective function defined as the sum of squared differences between EMG-driven and experimental $M_{\mathrm{KFE}}$. The algorithm stop criterion assumed that the difference between two consecutives objective functions had to be less than $10^{-3}$ for four function evaluations in a row. In this process, the EMG-driven model learned to reproduce $F^{m t}$ accounting for the different muscle EMG patterns that can produce the same walking or running motor task. It is worth stressing the fact that the elastic tendon and the infinitely stiff-tendon MTU models were calibrated separately starting from the same uncalibrated parameter set. This led to different optimal parameter sets for the two models. However, in this process, $l_{o}^{m}$ was kept the same across the two models.

\section{Validation Procedure}

The validation comprised of three tests. The first test compared the behavior of the SHM component to that of the EHM component. MTU forces and KFE moments estimated using the SHM and EHM components, and KFE moments obtained experimentally were time normalized during the stance phase using a cubic spline and averaged across all trials from all subjects. This produced ensemble average curves of the $F^{m t}$ and the $M_{\mathrm{KFE}}$ that were further compared using the square of the Pearson product moment correlation $\left(R^{2}\right)$ and the root mean squared error (RMSE) coefficients. Optimized values for the muscle activation and muscle-tendon parameters that were obtained for both the SHM and the EHM-based EMG-driven models were averaged across subjects and compared. To quantify how tendon length varied during the dynamic trials, the percent tendon strain $\% \varepsilon_{T}=100 \cdot \varepsilon_{T}$ was calculated using the EHM component. The $\% \varepsilon_{T}$ associated with each MTU was computed at each instant of time for all dynamic trials performed by all subjects. For each MTU, the average $\% \varepsilon_{T}$, with associated standard deviation, was calculated across subjects over the entire duration of each trial. To quantify the impact of using infinitely stiff tendons on MTU fiber length, we calculated how much the fiber length $l_{s}^{m}$ computed using the SHM component, deviated from the fiber length $l_{e}^{m}$ computed using the EHM component. The percentage length variation $\Delta_{L}$ was defined by $\Delta_{L}=\frac{l_{s}^{m}-l_{e}^{m}}{l_{e}^{m}} \times 100$.

The second test quantified how much the MTK component misfits contributed to the EMGdriven model $M_{\mathrm{KFE}}$ prediction error. In this analysis, moments $\left(M_{\mathrm{OSIM}}\right)$ calculated using OpenSim-generated $\ell^{\text {ht }}$ and $r$ values were compared to moments ( $M_{\mathrm{MTK}}$ ) obtained using MTK-generated $\not h t$ and $r$ values. $M_{\mathrm{OSIM}}$ moments were assumed to be our gold standard because the MTK spline coefficients were initially obtained from OpenSim-generated $\ell^{n t}$ nominal values (see Section II-C). $M_{\mathrm{MTK}}$ and $M_{\mathrm{OSIM}}$ were time normalized on all dynamic trials from all subjects during the stance phase. The percentage variation of $M_{\mathrm{MTK}}$ with respect to $M_{\mathrm{OSIM}}$ was then computed to finally quantify the impact on the EMG-driven model $M_{\mathrm{KFE}}$ prediction $\Delta_{M}=\frac{M_{\mathrm{MTK}}-M_{\mathrm{OSIM}}}{M_{\mathrm{OSIM}}} \times 100$.

In the third test, we computed the execution time for the individual SHM, EHM, and MTK components. Then, we quantified how the EMG-driven model computational time varied during calibration and open-loop execution, when using the SHM and the EHM

\footnotetext{
${ }^{5}$ Optimization parameters included the starting temperature $(T=20)$, the temperature reduction factor $(\mathrm{RT}=0.4)$, number of inner loops taken before temperature reduction ( $\mathrm{NT}=5$ ), number of steps in inner loop ( $\mathrm{NS}=20)$, and maximum number of objective function evaluations (MAXEVAL $=15000)$.
} 
components, respectively. Calibration time was calculated as the time needed to calibrate the EMG-driven model on the four calibration trials averaged across the calibration times individually obtained on the six subjects. Execution time was calculated as the average time needed to repeatedly compute one sample of the $M_{\mathrm{KFE}} 1000$ times. Tests were performed on an embedded system equipped with a 1.6-GHz CPU and 512-MB RAM.

\section{Results}

In the first test (see Fig. 3), $F^{m t}$ curves predicted by the SHM and the EHM components were very similar and highly correlated $\left(R^{2}>0.9\right)$ for MTUs including sm, st, bflh, bfsh, sar, rf, gra, vm, vi, and vl. For these, the SHM component predicted a slightly higher force contribution than the EHM component with exception of the bflh and rf. The mg forces predicted from the EHM and SHM components were well correlated $\left(R^{2}=0.71\right)$ and developed similar force amplitudes. Only for the tfl and for the lg MTUs the EHM and SHM predicted forces were poorly correlated $\left(R^{2} \leq 0.2\right)$, with the EHM component predicting a substantially higher force contribution than the SHM component.

The overall similar behavior of the EHM- and SHM-predicted $F^{m t}$ was justified by a similarity in the underlying optimized parameters. That is, starting with the same uncalibrated parameter set, the model calibration component (see Section II-C) produced two similar optimized parameter sets for both the SHM- and EHM-based models, respectively. For all subjects, the averaged EHM-calibrated $l_{S}^{t}$ values differed less than $1 \mathrm{~cm}$ from the averaged SHM-calibrated $l_{S}^{t}$ values for all MTUs with exception of the bflh, tfl, mg, and lg. For these MTUs, differences were between 2 and $4 \mathrm{~cm}$ (see Table I).

During all dynamic trials, the tendons assumed a close-to-stiff behavior. This is revealed in the $\% \varepsilon_{T}$ for all MTUs in the EHM component being always less than $2 \%$ [see Fig. 4(a)], Furthermore, the SHM-generated $P^{m}$ always varied less than 9\% compared to the EHMgenerated $P^{m}$ during all dynamic trials for all subjects [see Fig. 4(b)], revealing a low impact of the infinitely stiff tendon on the fiber length variation. Note that the same $l_{o}^{m}$ value was used for both SHM and EHM components (see Section II-C). Also, the global parameters calibrated for the EHM- and SHM-based EMG-driven models assumed substantially similar values. For the EHM- and SHM-based EMG-driven models, respectively, the flexor coefficient values were $0.8 \pm 0.5$ and $1.1 \pm 0.3$, the gastrocnemius coefficient values were $1.6 \pm 0.4$ and $1.7 \pm 0.9$, the extensor coefficient values were $1.6 \pm 0.5$ and $1.7 \pm 0.6$, the $C 1$ coefficient values were $-0.6 \pm 0.5$ and $-0.9 \pm 0.3$, the $C 2$ coefficient values were $-0.6 \pm 0.5$ and $-0.4 \pm 0.5$, and the shape factor values were $0.2 \pm 0.1$.

As a result, KFE moments predicted using the EHM and SHM components were very similar, being highly correlated $\left(R^{2}=0.89\right)$ [see Fig. 4(c)]. However, the SHM-predicted KFE moments were better correlated to the experimental $M_{\mathrm{KFE}}$ with respect to the EHMpredicted KFE moments. The SHM-predicted KFE moments had a $R^{2}$ coefficient of 0.93 with experimental $M_{\mathrm{KFE}}$. The RMSE was $7.89 \mathrm{~N} \cdot \mathrm{m}$, with the SHM component introducing an error that, on average, was less than the $10 \%$ of the range of variation observed in the reference values (i.e., $86.67 \mathrm{~N} \cdot \mathrm{m}$ ). The maximum absolute error $e_{\max }$ was equal to 21.49 $\mathrm{N} \cdot \mathrm{m}$. It occurred at $73 \%$ of the stance phase and was located toward the edge of the data due to inherent limitations in the filtering process at the end of data series. The minimum absolute error $e_{\min }$ was equal to $0.12 \mathrm{~N} \cdot \mathrm{m}$ and occurred at $47 \%$ that was the peak region. Compared to the SHM, the EHM $R^{2}$ coefficient and RMSEs with the experimental $M_{\mathrm{KFE}}$ assumed poorer values: $R^{2}=0.89, \mathrm{RMSE}=9.72 \mathrm{~N} \cdot \mathrm{m}$. However, minimum and maximum absolute errors provided better results: $e_{\max }=19.031 \mathrm{~N} \cdot \mathrm{m}$ and $e_{\min }=0.012 \mathrm{~N} \cdot \mathrm{m}$. 
In the second test (see Fig. 5), the MTK component fitting errors produced a percent variation in the predicted $M_{\mathrm{KFE}}$ that was always less than the $2.5 \%$ of the moments obtained using OpenSim-generated $\ell^{p t}$ and $r$.

Results from the third test revealed the SHM component executed in $0.07 \pm 0.00068 \mathrm{~ms}$, which is 250 times faster than the EHM component execution time, i.e., $17.5 \pm 0.072 \mathrm{~ms}$. The time needed by the MTK component to execute varied across MTUs as a function of the MTU's spanned DOFs [11]. It ranged from 0.001 (MTUs spanning one DOF) to $0.08 \mathrm{~ms}$ (MTUs spanning four DOFs). The time needed to predict the kinematics for all MTUs in the model was $1.1 \pm 0.043 \mathrm{~ms}$. The total execution time depended on the integration of the MTK with the SHM (Model(SHM,MTK)) or EHM (Model(EHM,MTK)) components. The Model(SHM,MTK) executed in $3.6 \pm 0.064 \mathrm{~ms}$, while the Model(EHM,MTK) needed 21.1 $\pm 0.11 \mathrm{~ms}$. Finally, the time to calibrate the Model(SHM,MTK) (158.84 s) was dramatically less than the time needed to calibrate the Model(EHM,MTK) (4 h and $48 \mathrm{~min}$ ).

\section{General Discussion}

This section further discusses the results presented in this study and highlights the related benefits and drawbacks.

\section{A. Model Prediction Ability}

The calibrated EMG-driven model is an open-loop system with $F^{m t}$ determined as a function of EMG linear envelopes and 3-D joint angles. During our experiments, the calibrated EMG-driven model was able to accurately predict muscle-generated joint moments from motor trials that were not used in the calibration process. This highlights the ability of our proposed methodology to predict muscle behavior and the resulting KFE moments.

\section{B. Infinitely Stiff Tendon}

Results showed that starting from the same uncalibrated parameter set, it was possible to obtain two similar optimized parameter sets for both the SHM and EHM-based models. The benefit is our proposed infinitely stiff MTU model allows substantially decreasing both the calibration time for the parameter identification, and the execution time with no loss of accuracy with respect to the elastic-tendon MTU model. Results on $F^{m t}$ prediction (see Fig. 3) showed that the SHM-based EMG-driven model produced substantially similar force estimations to those obtained by using a nonlinear elastic tendon with the only exception of the tfl and lg MTUs. For these two MTUs, differences in SHM and EHM-based $F^{m t}$ predictions may be directly associated with differences between SHM-calibrated and EHMcalibrated $l_{s}^{t}$ which were substantially high with respect to the other MTUs (see Table I). Results on joint moment prediction accuracy (see Fig. 4) showed that the SHM-based EMGdriven model was indeed better able to predict experimental KFE moments than when using the EHM component. This may be related to multiple causes and further work is needed to understand the underlying reason. However, it is important to stress the fact that results were obtained from walking and running tasks where tendons, in muscles crossing the knee joint, naturally assume a very stiff behavior. With these conditions, the elastic-tendon model we used in this study [1]-[3] might had been unable to replicate this very stiff tendon behavior while the approximation given by our proposed infinitely stiff-tendon model might had been closer to the true tendon behavior. A better insight into the compliance properties of human tendons might help design better tendon models with important implications for energetics that could not be properly addressed using the simplified stiff-tendon model presented in this paper. 
However, it is worth noting that our proposed MTU model accounts for the change in muscle fiber thickness during contraction as a function of muscle activation as opposed to the elastictendon-based MTU model in which muscle fiber thickness is kept constant (see Section II-C). In (3), muscle fiber thickness is related to $a(t)$ by using the muscle activationdependant $l_{o}^{m}$. This modeling approach may help representing physiological fiber contraction dynamics of muscles in which the actual fiber thickness is not constant during contraction [19]. This may further explain why the use of an infinitely stiff tendon model produced a substantial change in $F^{m t}$ for the tfl, lg, and bflh (see Fig. 3) even though these MTUs had a rather stiff tendon behavior to begin with [see Fig. 4(a)]. However, since similar net knee flexion-extension moments were predicted by both the EHM and SHM-based MTU models [see Fig. 4(c)], the observed changes resulted in a slightly different MTU force distribution strategy. Future studies will investigate whether these differences in the force distribution strategy may influence the EMG-driven model's ability of predicting joint moments on the frontal and coronal planes.

\section{MTK}

The method previously developed by the authors [11] was used to obtain accurate estimations of MTK (see Section II-C). For the first time, it was integrated in the proposed EMG-driven model and executed on an embedded system. Results showed that the MTK component did not affect the EMG-driven model computational time and prediction accuracy. That is, the overall $M_{\mathrm{KFE}}$ percentage variation and computational time associated with the MTK component were always less than $2.5 \%$ and $1.2 \mathrm{~ms}$, respectively.

\section{Computational Time}

Calibration time increases nonlinearly with the number of muscles in the model and with the number of calibration trials. The fast calibration that we obtained is therefore especially important when the EMG-driven model is to be scaled up to whole-body level and a large number of motor trials are to be used for calibration. Furthermore, our methodology permits implementing an online calibration. This could keep refining the EMG-driven model activation parameters as the model executes, thus accounting for parameters drift due to muscle fatigue or to a change in skin-electrode impedance.

\section{E. Limitations of Our Proposed Methodology}

The proposed EMG-driven model integrated in the embedded system still requires extra instrumentation to measure EMG and joint/segmental angles and moments. In our proposed study, input data were directly loaded on the embedded system internal memory, thus avoiding the need for using external sensors. Future work could use combined wireless EMG and six-DOF motion sensors for implementing a portable and real-time musculoskeletal analysis system [20].

Our proposed EMG-driven model uses EMG signals generated at an instant of time to predict the future muscle activation value $10 \mathrm{~ms}$ forward in time, i.e., after the EMD. Ideally, the model also requires real-time predictions of joint angles $10 \mathrm{~ms}$ forward in time to enable estimates of the corresponding MTK and the subsequent prediction of musculotendon forces and joint moments. External motion sensors cannot provide joint angle measurements forward in time. Future implementations could use full forward dynamics models [21] or nonparametric methods such as Bayesian filtering [22] to predict the real-time MTK within the EMD period, constantly updated using data from the aforementioned six-DOF motion sensors.

Our proposed methodology can produce reliable estimates of muscle behavior when the majority of EMGs are measured at a joint. When few EMGs are available, the model will 
allow muscles to work outside their physiological range of operation. As a result, muscles will most likely produce more force they are supposed to in order to make up for the missing muscles contribution. While this scenario is not ideal to perform investigations on physiological muscle behavior, it might represent a practical solution to achieve proportional torque-driven control of prostheses and orthoses joints using a limited number of EMG sensors as previously suggested [5]-[7]. Future work could investigate how the device control performances vary with the muscles included in the model.

\section{Conclusion}

In this paper, we presented a novel EMG-driven musculoskeletal model of the knee joint that can produce muscle force and moment estimates within the lower limb muscles EMD (i.e., $10 \mathrm{~ms}$ ) when operated on an embedded system with limited computational power. This fast operation was achieved while accounting for the 3-D kinematics of muscles and for the physiology of muscle fibers. We showed that, during walking and running, tendons could be modeled as infinitely stiff bodies with minor differences in the predicted MTU forces (see Fig. 3) and no loss of joint moment prediction accuracy with respect to the previously developed elastic-tendon model [see Fig. 4(c)].

In the context of the design of HMIs for myoelectrically controlled prostheses and orthoses, the joint moments predicted by our proposed methodology are more desirable than those predicted using the elastic-tendon model. This is because the SHM-generated moments closely match the experimentally measured moments, while doing this fast enough so they can be used in embedded controllers. Therefore, the SMH-based EMG-driven model can be used to produce reliable control signals for a torque-controlled assistive device, which permits device actuation to be synchronized to the person's muscle contraction in an intuitive and natural manner [5]-[7]. Finally, the availability of the proposed EMG-driven musculoskeletal model will facilitate the transition toward the design of human-inspired devices that can effectively take advantage of a person's own neural control of movement without relying on explicit representations of the subject's task-specific neural control models.

\section{Acknowledgments}

This work was supported in part by the National Institutes of Health, U.S., under Grant R01EB009351-01A2, the National Health and Medical Research Council, Australia, under Grant 628850 and Grant 334151, the Western Australia Medical and Health Research Infrastructure Council, the CARIPARO Foundation, Italy, under Grant "Progetto Dottorati di Ricerca 2008-2010," and the University of Padova under Grant "Progetto di Ateneo 20102011."

\section{Biographies}

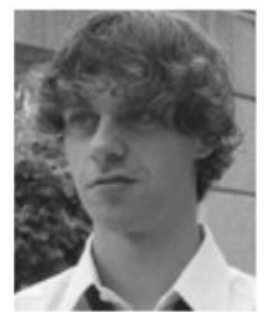

Massimo Sartori (M'09) received the B.E. and M.S. degrees in computer engineering from the University of Padova, Padova, Italy, in 2004 and 2007, respectively, where he received the Ph.D. degree from the Graduate School on Information Engineering in 2011. 
During his Ph.D., he visited the School of Sport Science, Exercise and Health, University of Western Australia, and the Neuromuscular Biomechanics Lab, Stanford University, Stanford. He then was with the Griffith Health Institute, Griffith University, Brisbane, Australia. His main research interests include neuromusculoskeletal modeling, neuromuscular human-machine interfaces, and assistive device control.

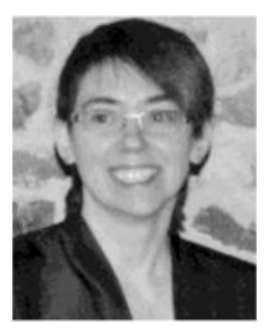

Monica Reggiani (M'98) received the Graduate and Ph.D. degrees in computer engineering from the University of Parma, Parma, Italy in 1997 and 2001, respectively.

During 1999-2000, she was a Visiting Scholar at the Los Alamos National Laboratory. She was a Research Associate in the Dipartimento di Ingegneria dell'Informazione, University of Parma in 2005 and a Research Associate in the European project Xpero with the University of Verona during 2006-2007. She was a Visiting Professor at the University of Applied Sciences Bonn-Rhein-Sieg in 2007 and a Visiting Researcher at the University of Western Australia in 2010. She is currently an Assistant Professor at the University of Padova, Padova, Italy. Her research interests include human-robot interfaces, motion planning, programming by demonstration, and autonomous robots.

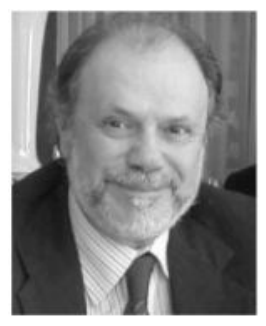

Enrico Pagello received the Laurea degree in electronic engineering from the University of Padova, Padova, Italy, in 1972.

From 1976 to 1983, he was at the Institute of Biomedical Engineering, National Research Council, Padova, where he is currently a part-time Senior Associated Researcher. During 1977-1978, he was a Visiting Scholar in the Artificial Intelligence Laboratory, Stanford University, Stanford, CA. Since 1983, he has been a Professor of computer science at the University of Padova. He was an Invited Professor at Keio University between 2005 and 2008 and was elected a Fellow of the University of Tokyo in 2009.

Mr. Pagello was a General Chair of the 6th International Conference on Intelligent Autonomous Systems in 2000, 7th Robot World Cup Soccer and Rescue Competitions in 2003, and 1st International Conference on Simulation, Modeling, and Programming for Autonomous Robots in 2008, and a member of the IEEE Transactions on Robotics and Automation Editorial Board. He is currently in the IEEE Robotics and Automation Society International Journal Editorial Board and has been the President of the International Intelligent Autonomous Systems Society since 2004. 


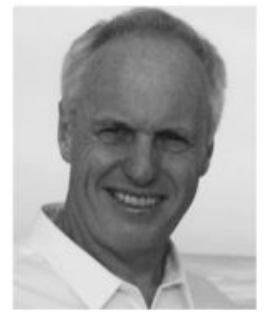

David G. Lloyd received the B.Sc. degree in mechanical engineering from the University of New South Wales, Sydney, Australia, in 1984. He received the Ph.D. degree in biomechanical engineering from the University of New South Wales, Sydney, N.S.W., Australia, in 1994, after working in industry for several years.

He then had a postdoctoral training in neurophysiology and computational biomechanics in the Sensory Motor Performance Program, Northwestern University, Chicago, IL. He is currently a Professor and the Director of the Centre for Musculoskeletal Research, Griffith University, Brisbane, Qld., Australia, with adjunct Professorial appointments at the University of Western Australia and the University of Delaware. His research is focused on neuromuscular skeletal modeling to understand causes and treatments for musculoskeletal disease and injury.

\section{References}

[1]. Winby CR, Lloyd DG, Besier TF, Kirk TB. Muscle and external load contribution to knee joint contact loads during normal gait. J. Biomech. 2009; 42(14):2294-2300. [PubMed: 19647257]

[2]. Lloyd DG, Besier TF. An EMG-driven musculoskeletal model to estimate muscle forces and knee joint moments in vivo. J. Biomech. 2003; 36(6):765-776. [PubMed: 12742444]

[3]. Buchanan TS, Lloyd DG, Manal K, Besier TF. Estimation of muscle forces and joint moments using a forward-inverse dynamics model. Med. Sci. Sports Exerc. Nov; 2005 37(11):1911-1916. [PubMed: 16286861]

[4]. Pfeifer, S.; Hardegger, M.; Vallery, H.; List, R.; Foresti, M. Model-based estimation of active knee stiffness; Proc. IEEE Int. Conf. Rehab. Robot.; Jun./Jul. 2011; p. 909-914.

[5]. Cavallaro EE, Rosen J, Perry JC, Burns S. Real-time myoprocessors for a neural controlled powered exoskeleton arm. IEEE Trans. Biomed. Eng. Nov; 2006 53(11):2387-2396. [PubMed: 17073345]

[6]. Ferris DP, Gordon KE, Sawicki GS, Peethambaran A. An improved powered ankle-foot orthosis using proportional myoelectric control. Gait Posture. 2006; 23(4):425-428. [PubMed: 16098749]

[7]. Fleischer C, Hommel G. A human-exoskeleton interface utilizing electromyography. IEEE Trans. Robot. Aug; 2008 24(4):827-882.

[8]. Sartori, M.; Reggiani, M.; Lloyd, DG.; Pagello, E. A neuromusculoskeletal model of the human lower limb: Towards EMG-driven actuation of multiple joints in powered orthoses; Proc. Int. Conf. Rehab. Robot.; 2011. p. 709-714.

[9]. Sartori, M.; Lloyd, DG.; Reggiani, M.; Pagello, E. Fast operation of anatomical and stiff tendon neuromuscular models in EMG-driven modeling; Proc. IEEE Int. Conf. Robot. Autom.; May 2010; p. 2228-2234.

[10]. Zajac FE. Muscle and tendon: Properties, models, scaling, and application to biomechanics and motor control. Crit. Rev. Biomed. Eng. 1989; 17(4):359-411. [PubMed: 2676342]

[11]. Sartori M, Reggiani M, van den Bogert AJ, Lloyd DG. Estimation of musculotendon kinematics in large musculoskeletal models using multidimensional B-splines. J. Biomech. 2012; 45(3):595601. [PubMed: 22176708]

[12]. Nordez A, Gallot T, Catheline S, Guevel A, Cornu C, Hug F. Electromechanical delay revisited using very high frame rate ultrasound. J. Appl. Physiol. Jun; 2009 106(6):1970-1975. [PubMed: 19359617] 
[13]. Winter, DA. Biomechanics and Motor Control of Human Movement. Wiley; New York: 2004. p. 344

[14]. Delp SL, Anderson FC, Arnold AS, Loan P, Habib A, John CT, Guendelman E, Thelen DG. OpenSim: Open-source software to create and analyze dynamic simulations of movement. IEEE Trans. Biomed. Eng. Nov; 2007 54(11):1940-1950. [PubMed: 18018689]

[15]. Delp SL, Loan JP, Hoy MG, Zajac FE, Topp EL, Rosen JM. An interactive graphics-based model of the lower extremity to study orthopaedic surgical procedures. IEEE Trans. Biomed. Eng. Aug; 1990 37(8):757-767. [PubMed: 2210784]

[16]. Loan, JP. Dynamics Pipeline. Musculographics Inc.; Evanston, IL: 1992.

[17]. Huijing PA. Important experimental factors for skeletal muscle modelling: Non-linear changes of muscle length force characteristics as a function of degree of activity. Eur. J. Morphol. 1996; 34(1):47-54. [PubMed: 8743098]

[18]. Winby CR, Lloyd DG, Kirk TB. Evaluation of different analytical methods for subject-specific scaling of musculotendon parameters. J. Biomech. 2008; 41(8):1682-1688. [PubMed: 18456272]

[19]. Azizi E, Brainerd EL, Roberts TJ. Variable gearing in pennate muscles. Proc. Natl. Acad. Sci. U.S.A. Feb 5; 2008 105(5):1745-1750. [PubMed: 18230734]

[20]. Jovanov E, Milenkovic A, Otto C, de Groen PC. A wireless body area network of intelligent motion sensors for computer assisted physical rehabilitation. J. Neuroeng. Rehabil. Mar 1.2005 2(1)

[21]. Barrett RS, Besier TF, Lloyd DG. Individual muscle contributions to the swing phase of gait: An EMG based forward dynamics model. Simul. Modelling Pract. Theory. 2007; 15(9):1146-1155.

[22]. Ko J, Fox D. GP-BayesFilters: Bayesian filtering using Gaussian process prediction and observation models. Autonom. Robots. 2009; 27(1):75-90. 


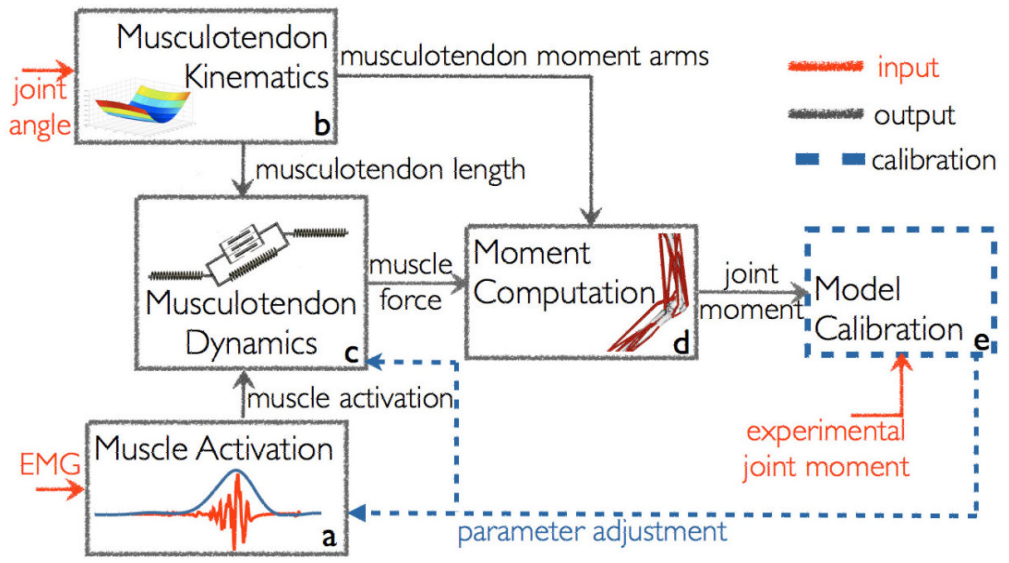

Fig. 1.

Schematic view of the EMG-driven model structure. RRA-generated experimental joint moments are used during the model calibration only (see Section II-C). After calibration, the model only needs EMG signals and IK-generated joint angles to determine muscle force and the resulting muscle-generated joint moments. 


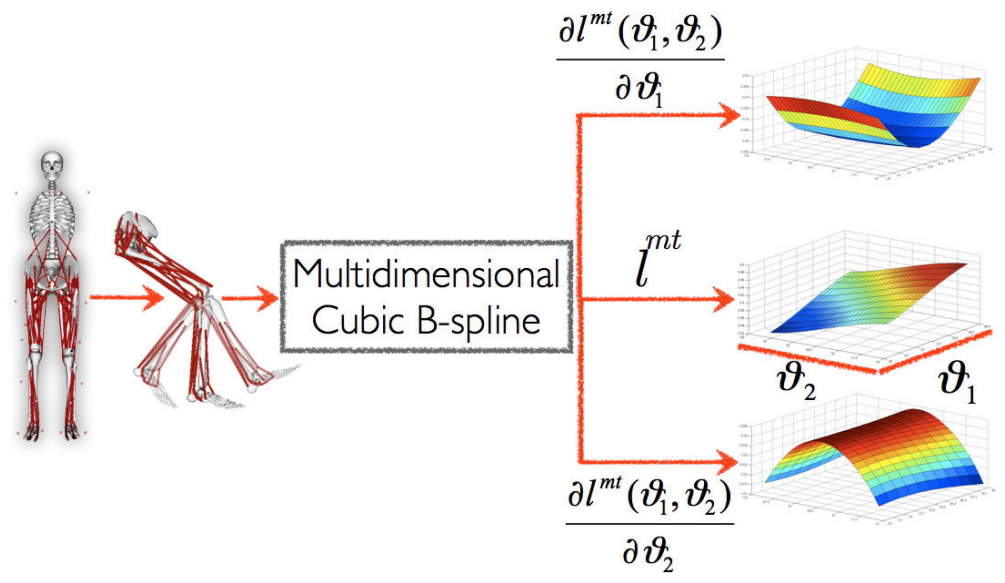

Fig. 2.

Scaled musculoskeletal model is used to obtain musculotendon length $\ell^{\text {pt }}$ nominal values for each muscle corresponding to discrete combinations of lower limb joint angles $\vartheta$. The $\not n t$ nominal values are then used to compute the coefficients for a multidimensional cubic spline function per muscle which is used to generate $\not h t$ values as a function of joint angles.

Moment arm values as a function of joint angles are obtained by differentiating the $\ell^{n t}$ spline function with respect to the joint angle of interest. 


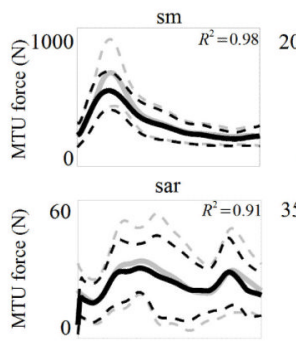

$\mathrm{vm}$

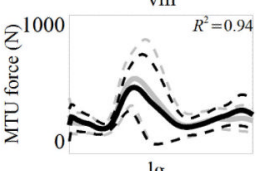

$\lg$

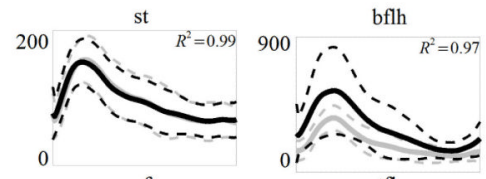

rf

tfl

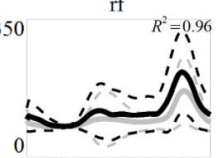

900
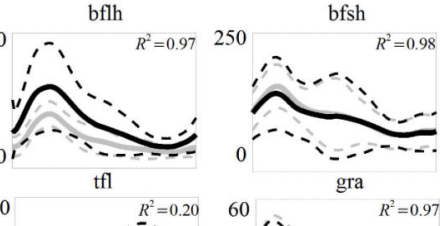

gra
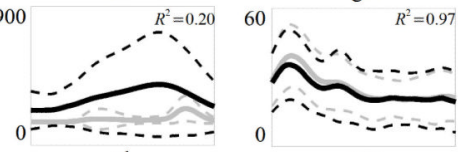

$\mathrm{vl}$

vi

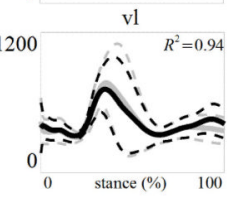

000
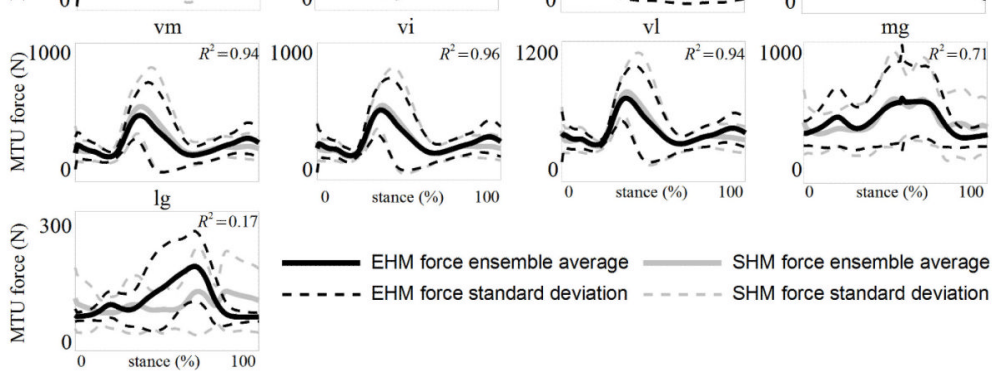

SHM force ensemble average SHM force standard deviation

Fig. 3.

First test. The MTU force estimated using the elastic-tendon model (EHM) and the infinitely stiff-tendon model (SHM) is compared for all MTUs as described in Section III. The square of the Pearson product moment correlation coefficient $\left(R^{2}\right)$ between EHM and SHM force is reported for each MTU where names are defined as in Section II-C. 

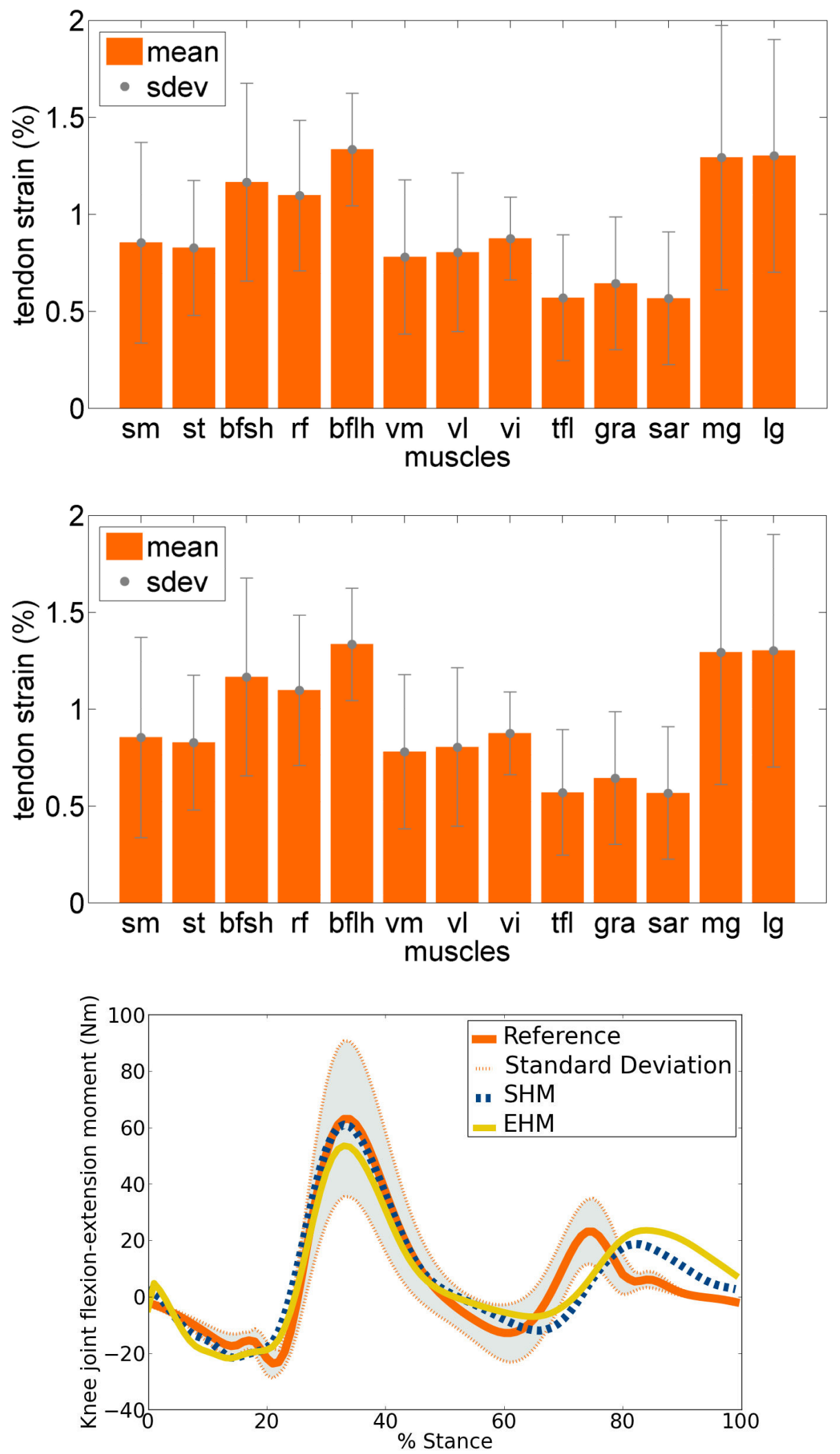

Fig. 4. 
First test. Values of (a) tendon strain and (b) fiber length variation averaged over the entire duration of all dynamic trials for all subjects. (c) Ensemble average of the experimental KFE moment (reference) is compared to that estimated using the infinitely stiff-tendon muscle model (SHM) and to that estimated using the elastic-tendon muscle model (EHM). The standard deviation of the ensemble average of the reference KFE moment is represented by the dotted lines and the shaded area. Reference moments were obtained using RRA (see Section II-B). MTU names are defined as in Section II-C. 


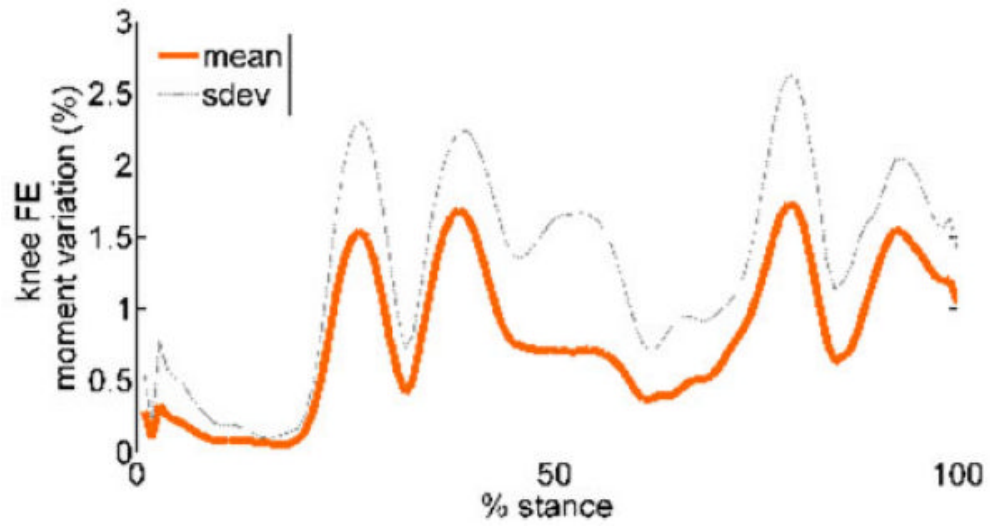

Fig. 5.

Second test. The percentage variation of the KFE moments is estimated as described in Section III. The upper standard deviation is graphed to quantify the maximum KFE moment variation. 


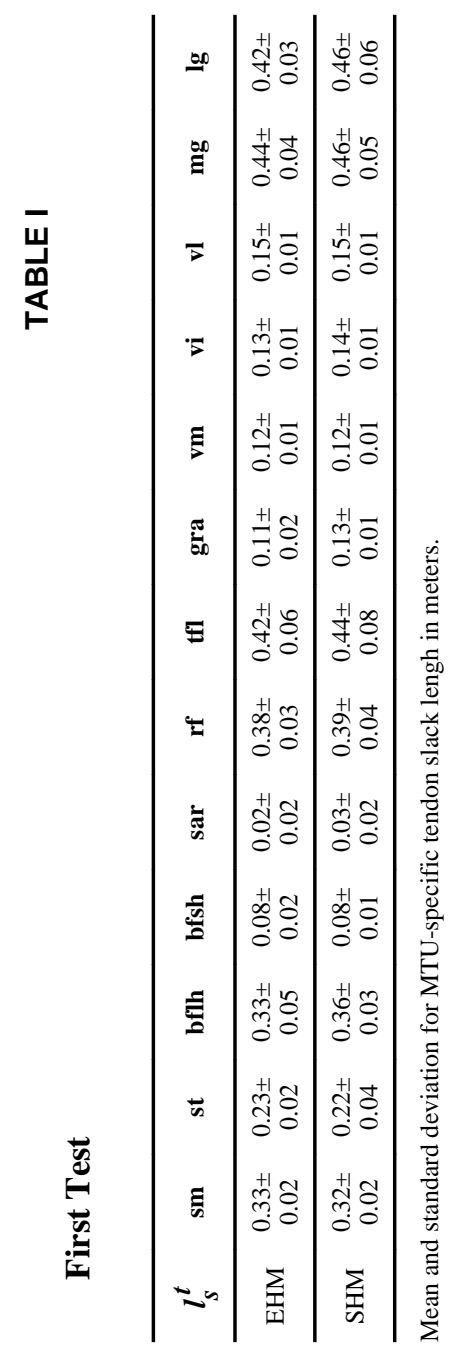

IEEE Trans Biomed Eng. Author manuscript; available in PMC 2013 September 01. 